\title{
Wet wrap dressings as a rescue therapy option for erythrodermic psoriasis*
}

\author{
Beatriz Regina de Fatima Navrotski ${ }^{1}$, Francielle Melina Nihi ${ }^{1}$, Michael Joseph Camilleri ${ }^{2}$, \\ Felipe Bochnia Cerci ${ }^{1}$
}

DOI: http:/ / dx.doi.org/10.1590/abd1806-4841.20186414

\begin{abstract}
Wet wrap dressings provide an ancillary treatment option for erythrodermic patients. Wet wrap therapy consists of the application of topical corticosteroids on all affected surfaces, followed by the application of a warm moist cotton cloth with a dry cotton cloth on top. The advantages of this procedure include its safety, as well as the prompt and marked improvement of the erythroderma. For erythrodermic psoriasis, wet wrap dressings can serve as an important rescue therapy option. The purpose of this study is to report a case series of seven patients with erythrodermic psoriasis that responded promptly to wet wrap therapy prior to the initiation of systemic treatment.
\end{abstract}

Keywords: Administration, topical; Corticosteroids; Glucocorticoids; Psoriasis; Therapeutics

Erythroderma is defined as an inflammatory dermatosis that involves more than $90 \%$ of the body surface area. The most common cause is psoriasis. ${ }^{1,2}$ Prompt improvement of erythrodermic psoriasis is necessary to avoid complications such as water-electrolyte imbalance and infections. ${ }^{1}$ However, systemic treatments take days to weeks to achieve their full effect. ${ }^{2}$

Wet wrap therapy (WWT) is classically described as a rescue therapy option for atopic dermatitis, but it has also been reported for the treatment of psoriasis. ${ }^{1,3-5}$ It consists of the application of topical corticosteroids or emollient on all affected surfaces, followed by application of a warm moist cotton cloth with a dry cotton cloth on top. ${ }^{3,6}$

This study was approved by the Institutional Review Board of the Pontifícia Universidade Católica do Paraná, Brazil. We report a case series of seven patients with erythrodermic psoriasis who responded promptly to WWT.

CASE 1: Male, 62 years old, with plaque psoriasis, on topical treatment. He developed erythrodermic psoriasis after using oral terbinafine for tinea cruris (Figure 1).
CASE 2: Male, 76 years old, with plaque psoriasis, using acitretin with partial control. He developed erythrodermic psoriasis without a known etiology.

CASE 3: Male, 44 years old, with erythrodermic psoriasis using oral prednisone without dermatology follow-up. He had a flare after discontinuation of prednisone.

CASE 4: Female, 26 years old, with plaque psoriasis since childhood, using oral prednisone without follow-up for more than 8 years. She developed erythrodermic psoriasis without a known etiology.

CASE 5: Female, 23 years old, with plaque psoriasis and atopic dermatitis, using topical corticosteroid and under irregular follow-up with dermatology, resistant to cyclosporine use. She developed erythrodermic psoriasis while on topical treatment.

CASE 6: Female, 38 years old, with plaque psoriasis and psoriatic arthritis using infliximab, which was discontinued after she was diagnosed with non-Hodgkin's lymphoma, leading to the development of erythrodermic psoriasis.

\footnotetext{
Received 24 August 2016.

Accepted 10 October 2017.

* Work conducted at the Hospital Santa Casa de Curitiba, Curitiba (PR), Brazil. Financial Support: None.

Conflict of Interest: None.

1 Dermatology Service, Hospital Santa Casa de Curitiba, Curitiba (PR), Brazil. Department of Dermatology, Mayo Clinic, Rochester, MN, USA.
}

MaILING AdDREsS:

Beatriz Regina de Fatima Navrotski

E-mail: biantk@hotmail.com

C2018 by Anais Brasileiros de Dermatologia 
CASE 7: Male, 69 years old, without previous history of psoriasis, developed erythrodermic psoriasis confirmed by histology.

All patients were admitted to hospital for treatment. While in hospital, patients were submitted to WWT consisting of application of topical corticosteroids (medium to high potency) on all affected surfaces, two to four times a day for one hour each application. All patients showed marked improvement after 48 to 72 hours. As maintenance therapy, patients were managed with PUVA, narrowband UVB, cyclosporine, cyclosporine with acitretin, or ustekinumab with adequate long-term control. The follow-up period in these patients ranged from 15 to 33 months (mean: 24 months). No recurrence of erythroderma was observed in any of the patients during follow-up.

Erythrodermic psoriasis can occur abruptly, leading to serious systemic complications. In such cases, hospitalization with supportive measures and systemic therapies must be considered for fast disease control. ${ }^{1,2}$

First-line therapy for severe cases consists of cyclosporine or infliximab. However, even these drugs may take days to weeks for adequate control of erythroderma. ${ }^{1,2}$ The use of topical corticosteroids through WWT provides pain relief, reduces itching, and restores the skin barrier, speeding up recovery of these patients even before systemic therapy starts taking effect. 5,6,7

Several WWT protocols have been described, usually with weak or medium-strength topical corticosteroids and long application duration ( 3 hours each).-9 Our protocol includes the use of medium to high-potency corticosteroid creams, (betamethasone dipropionate $0.05 \%$ or clobetasol dipropionate $0.05 \%$ ) on all affected surfaces, followed by application of a warm moist cotton cloth with dry cotton cloth on top (Figure 2A). Bedsheets or blankets may be used instead of dry cotton cloth to avoid heat loss and to retain heat from the moist cotton cloth (Figure 2B). When available, ambient heating should be used to improve patient comfort. WWT is performed two to four times a day for one hour each application (during daytime). It is important to emphasize that WWT must be used for only a few days ( 2 to 5 maximum), due to the side effects of topical corticosteroids. As the scaling and erythema improve, previously affected areas should be treated with emollients. ${ }^{5}$
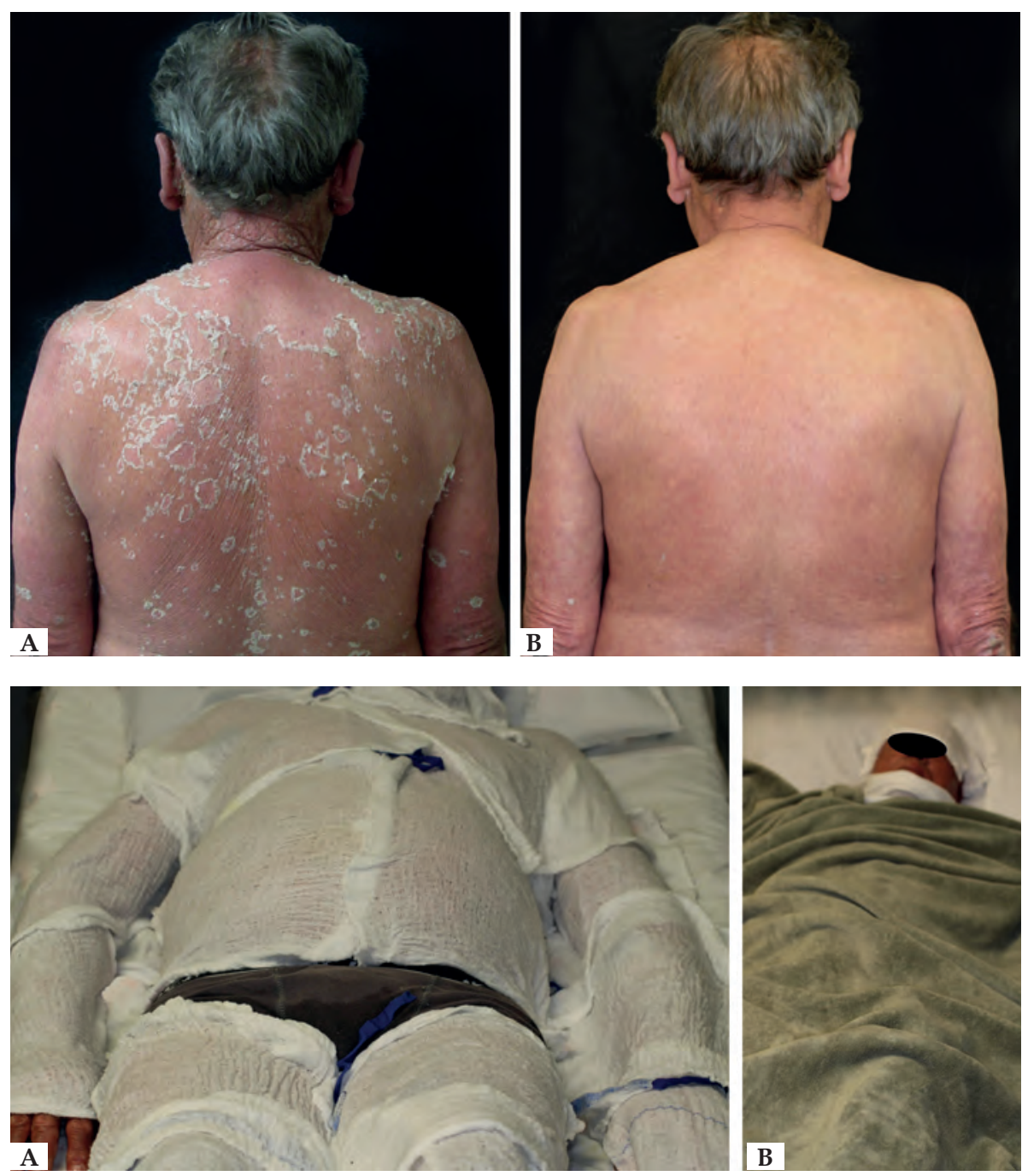

FIGURE 2: A - Application of warm moist cotton cloth on top of topical corticosteroids. Dry cotton cloth is placed over these two.

B - Bedsheets or blankets can be used on top or instead of dry cotton cloth to avoid heat loss and to retain heat from the moist cotton cloth, thereby improving patient comfort 
The improvement in itching, erythema, and scaling in the seven cases was dramatic. After only 24 hours of therapy, patients reported substantial improvement of symptoms and quality of sleep.

The advantages of WWT include its relatively low cost, safety, and prompt response, leading to shorter length of hospital stay and lower risk of complications associated with erythrodermic psoriasis.

Although WWT has no specific contraindications, it should be avoided in patients with active systemic infections. ${ }^{4}$ Adverse events are rare and include folliculitis, skin atrophy, and herpes simplex infection. ${ }^{7}$ WWT safety has been well documented when used for a short period of time. Furthermore, no prolonged hypo- thalamic-pituitary-adrenal axis suppression was noted in previous studies. ${ }^{5}$ There are reports of patients with transient decreases in morning cortisol levels during treatment, but levels normalized after 2 weeks. ${ }^{3,10}$

The main limitation of this study is the small number of patients. Few articles have referred to WWT for erythrodermic psoriasis, and to our knowledge there have been no publications on the subject in the Brazilian literature. ${ }^{5,6}$

WWT should be considered as rescue therapy for erythrodermic psoriasis. It is important to emphasize that it should only be used for a few days. Systemic treatment should be introduced as soon as possible for long-term disease control. ${ }^{1,2} \square$

\section{REFERENCES}

1. Sociedade Brasileira de Dermatologia. Consenso Brasileiro de psoríase 2012 Guias de avaliação e tratamento. Rio de Janeiro: Sociedade Brasileira de Dermatologia; 2012. p. 2: 160-62.

2. Rosenbach M, Hsu S, Korman NJ, Lebwohl MG, Young M, Bebo BF Jr, et al Treatment of erythrodermic psoriasis: from the medical board of the National Psoriasis Foundation. J Am Acad Dermatol. 2010;62:655-62.

3. Devillers AC, Oranje AP. Efficacy and safety of wet-wrap dressings as an intervention treatment in children with severe and/or refractory atopic dermatitis: a critical review of literature. Br J Dermatol. 2006;154:579-85.

4. Janmohamed SR, Oranje AP, Devillers AC, Rizopoulos D, van Praag MC, Van Gysel D, et al. The proactive wet-wrap method with diluted corticosteroids versus emollients in children with atopic dermatitis: a prospective, randomized, doubleblind, placebo-controlled trial. J Am Acad Dermatol. 2014;70:1076-82.

5. Bingham LG, Noble JW, Davis MD. Wet dressings used with topical corticosteroids for pruritic dermatoses: A retrospective study. J Am Acad Dermatol. 2009;60:792-800.
6. Andersen RM, Thyssen JP, Maibach HI. The role of wet wrap therapy in skin disorders - a literature review. Acta Derm Venereol. 2015;95:933-9.

7. Dabade TS, Davis DM, Wetter DA, Hand JL, McEvoy MT, Pittelkow MR, et al. Wet dressing therapy in conjunction with topical corticosteroids is effective for rapid control of severe pediatric atopic dermatitis: experience with 218 patients over 30 years at Mayo Clinic. J Am Acad Dermatol. 2012;67:100-6.

8. Pei AY, Chan HH, Ho KM. The effectiveness of wet-wrap dressings using $0.1 \%$ mometasone furoate and $0.005 \%$ fluticasone proprionate ointments in the treatment of moderate to severe atopic dermatitis in children. Pediatr Dermatol. 2001;18:343-8.

9. Wolkerstorfer A, Visser RL, De Waard van der Spek FB, Mulder PG, Oranje AP Efficacy and safety of wet-wrap dressings in children with severe atopic dermatitis: influence of corticosteroid dilution. Br J Dermatol. 2000;143:999-1004.

10. Goodyear HM, Spowart K, Harper Jl. 'Wet-wrap' dressings for the treatment of atopic eczema in children. Br J Dermatol. 1991;125:604.

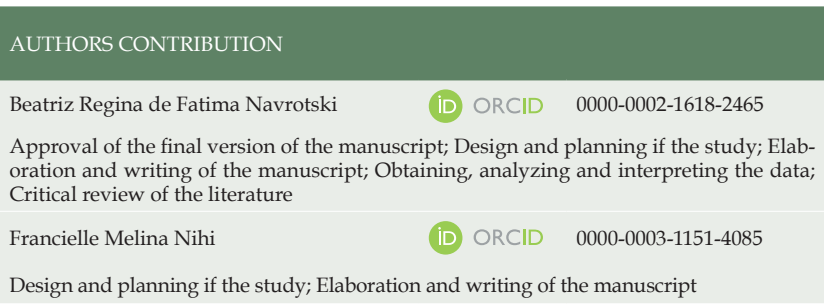

Michael Joseph Camilleri
Critical review of the literature; Critical review of the manuscript
Felipe Bochnia Cerci
$\begin{aligned} & \text { Approval of the final version of the manuscript; Design and planning if the study; Elab- } \\ & \text { oration and writing of the manuscript; Obtaining, analyzing and interpreting the data; } \\ & \text { Effective participation in research orientation; Critical review of the manuscript }\end{aligned}$

How to cite this article: Navrotski BRF, Nihi FM, Camilleri MJ, Cerci FB. Wet wrap therapy as a rescue therapeutic option for erythrodermic psoriasis. An Bras Dermatol. 2018;93(4):598-600. 